\title{
Data Mining of Prognostic Microenvironment-Related Genes in Clear Cell Renal Cell Carcinoma: A Study with TCGA Database
}

\author{
Bin Chen, Wei Chen, Jing Jin, Xueping Wang, Yifang Cao $\mathbb{D}$, and Yi He \\ Urology Department, Jiaxing First Hospital, First Affiliated Hospital of Jiaxing University, Jiaxing, Zhenjiang 314001, China \\ Correspondence should be addressed to Yifang Cao; caoyifang3301@sohu.com and Yi He; 84748480@qq.com
}

Received 18 July 2019; Revised 8 September 2019; Accepted 27 September 2019; Published 30 October 2019

Academic Editor: Marcello Ciaccio

Copyright (@ 2019 Bin Chen et al. This is an open access article distributed under the Creative Commons Attribution License, which permits unrestricted use, distribution, and reproduction in any medium, provided the original work is properly cited.

\begin{abstract}
Clear cell renal cell carcinoma (ccRCC) is one of the most prevalent kidney malignancies. The tumor microenvironment (TME) is highly related to the oncogenesis, progress, and prognosis of ccRCC. The aim of this study was to infer the level of infiltrating stromal and immune cells and assess the prognostic value of them. The gene expression profile was obtained from TCGA and used for calculating the stromal and immune scores. Based on a cut-off value, patients were divided into low- and highstromal/immune score groups. Survival analysis was performed to evaluate the prognostic value of stromal and immune scores. Moreover, differentially expressed genes (DEGs) that are highly related to TME were determined and applied for functional enrichment analysis and protein-protein interaction (PPI) network. The Kaplan-Meier plot demonstrated that patients with high-immune scores and stromal scores had poorer clinical outcome. In addition, a total of 89 DEGs were identified and mainly involved in 5 pathways. The top 5 degree genes were extracted from the PPI network; among them, IL10 and XCR1 were highly associated with prognosis of ccRCC. The results of the present study demonstrated that ESTIMATE algorithm-based stromal and immune scores may be a credible indicator of cancer prognosis and IL10 along with XCR1 may be a potential key regulator for the TME of ccRCC.
\end{abstract}

\section{Introduction}

Renal cell carcinoma (RCC) is the most prevalent kidney malignant tumor globally [1], and it is estimated that over 350,000 cases are diagnosed with RCC each year [2]. Clear cell renal cell carcinoma (ccRCC) is the most common and invasive form in all RCC and comprises about $70-80 \%$ of all RCC cases $[3,4]$.

In recent years, the tumor microenvironment (TME) has already attracted a huge amount of interest from researchers. TME is a complicated system which consists of an extracellular matrix, stromal cells (like fibroblasts, occasionally adipocytes, and mesenchymal stromal cells), and immune cells (such as B and T lymphocytes, macrophages, and natural killer cells) [5]. Immune and stromal cells are the most important component of nontumor cells in TME and have shown a potential value for diagnosis and prognosis prediction of cancers $[6,7]$. Previous studies have found that the extent of stromal cells could provide a prognostic factor for patients with solid cancers [8]. In addition, it also has been reported that activated CD8+ T cell density in TME is associated with favorable clinical outcomes of ccRCC $[9,10]$. Nevertheless, several immune cells have the opposite effect. For example, the recruitment of CD4+ T cells in TME could promote RCC proliferation through modulating TGF $\beta 1 / \mathrm{YBX} 1 /$ HIF2 $\alpha$ signals [11]. Moreover, regulatory T cells (Tregs) can also inhibit tumor immune responses by releasing immunosuppressive cytokines [12]. Macrophages have been reported to have crucial function in both promoting and blocking cancer growth. Macrophage M2 presenting CD163 and CD204 is highly associated with poor prognosis of RCC [13], whereas Hutterer et al. found that tumor-associated macrophages could independently reduce the risk of death in RCC [14].

Immunohistochemistry (IHC) and flow cytometry are the most commonly used technology for determining immune and stromal cells in TME by detecting marker proteins [15]. However, due to the restriction of the channel of markers, conventional technology could not evaluate diverse immune cells simultaneously [16]. As an alternative, algorithms based on a large scale of gene expression profile have 
been applied for predicting tumor purity in many researches [17]. The ESTIMATE (Estimation of Stromal and Immune cells in Malignant Tumor tissues using Expression data) algorithm developed by Yoshihara et al. is a new tool for inferring the level of infiltrating stromal and immune cells by calculating stromal and immune scores [17]. Subsequent researches on glioblastoma [18], colon cancer [19], and breast cancer [20] have been investigated with the ESTIMATE algorithm and shown good effectiveness of this algorithm. Nevertheless, research on ccRCC using the ESTIMATE algorithm has not been reported in detail.

In the present retrospective study, we applied the ESTIMATE algorithm for the analysis of gene expression profiles of ccRCC which were collected from The Cancer Genome Atlas (TCGA, https://cancergenome.nih.gov) to infer stromal and immune scores for the first time. The association of stromal and immune scores with clinicopathological parameter as well as clinical prognosis of ccRCC patients was also investigated.

\section{Materials and Methods}

2.1. Data Profile. The gene expression profiles of ccRCC were downloaded from TCGA (Apr 2019) and then were subjected to background correction and normalization with Perl 5.0 (http://www.perl.org/). Meanwhile, relevant clinical characteristics of cancer cases were also collected. Patients with follow-up time $<30$ days or lacking pathologic diagnosis would be removed.

2.2. ESTIMATE Algorithm. As described previously [17], with the ESTIMATE package in $\mathrm{R}$ (version 3.5.2, https:// www.r-project.org), stromal and immune scores of each sample were calculated. The optimal cut-off values were evaluated with the online tool: Cutoff Finder (http:// molpath.charite.de/cutoff/assign.jsp) [21]. Based on the cut-off values, patients were divided into low- and highstromal/immune score groups. Group comparisons of stro$\mathrm{mal} / \mathrm{imm}$ une scores between different clinical indexes were performed by the $t$-test with SPSS 20.0. $P$ value $<0.05$ was considered statistically significantly different.

2.3. Expression Analysis of Differentially Expressed Genes (DEGs). The Bioconductor package, "edgeR" (http://www .bioconductor.org/packages/release/bioc/html/edgeR.html) was utilized to identify DEGs between low- and high-stromal/immune score groups. The overlapping DEGs would be used for further analysis.

\subsection{Functional Enrichment Analysis and Protein-Protein} Interaction (PPI) Network. All overlapping DEGs were utilized for Kyoto Encyclopedia of Genes and Genomes (KEGG) and Gene Ontology (GO) analysis with $P$ value (adjusted $P$ value) $<0.05$ as the threshold. In addition, the PPI network of overlapping DEGs would be obtained from STRING (https://string-db.org) [22] with confidence $>0.7$ as a cut-off criterion. Then, the data of the PPI network was reconstructed by Cytoscape (version 3.6, https://cytoscape .org), and the top five degree DEGs were selected as the most important targets. The relationship between hub genes and
TABLE 1: The baseline characteristics of all patients.

\begin{tabular}{lccc}
\hline Parameter & Subtype & No. & Percent (\%) \\
\hline \multirow{2}{*}{ Age (years) } & $<65$ & 250 & 48.5 \\
\multirow{2}{*}{ Gender } & $\geq 65$ & 265 & 51.6 \\
& Male & 341 & 66.2 \\
TNM staging & Female & 174 & 33.8 \\
& Stage i/ii & 315 & 61.2 \\
Fuhrman grade & Stage iii/iv & 200 & 38.8 \\
& G 1/2 & 235 & 45.6 \\
Immune score & G 3/4 & 280 & 54.4 \\
\multirow{2}{*}{ Stromal score } & Low & 296 & 57.5 \\
& High & 219 & 42.5 \\
Survival status & Low & 317 & 61.6 \\
\multirow{2}{*}{ Total } & High & 198 & 38.4 \\
\hline
\end{tabular}

tumor-infiltrating immune cells (B cell, CD4 T cell, CD8 T cell, neutrophil, macrophage, and dendritic cell) was deciphered using TIMER (Tumor IMmune Estimation Resource, https://cistrome.shinyapps.io/timer/).

2.5. Survival Analysis. The Kaplan-Meier plots were conducted to illustrate survival difference between the low- and high-stromal/immune score groups with overall survival of ccRCC patients. Univariable and multivariable Cox regression model was used to determine independent prognostic factors. The $P$ value $<0.05$ was set as the cut-off value.

2.6. Expression Profile of Immunomodulators. In recent years, immune checkpoint inhibitors have been approved for the therapy of various cancers, including renal cell carcinoma [23]. In this study, several key immunomodulators (LAG-3, TIM-3, CTLA-4, IFN- $\gamma$, ICOS, ICAM-1, TIGIT, PD-1, PDL-1, NKG2A, and VISTA) were quantified in both normal kidney tissues and ccRCC tissues. The difference of expression of immunomodulators between normal and ccRCC samples as well as low- and high-stromal/immune score groups was compared by the $t$-test.

\section{Results}

3.1. Patient Characteristics. After removing cases with followup time $<30$ days, a total of 587 samples (72 normal kidney tissue samples and 515 ccRCC tissue samples) were collected from TCGA. The detailed demographic and baseline characteristics of 515 ccRCC patients are described in Table 1. All patients were diagnosed with ccRCC pathologically.

3.2. Evaluation of Immune Scores and Stromal Scores. Based on the cut-off values for stromal scores (841) or immune scores (1780), patients were assigned to low- and high-stromal/immune score groups. As shown in Figure 1(a), immune scores in Fuhrman grade $3 / 4$ were significantly increased compared with those in Fuhrman grade $1 / 2(P<0.001)$. In addition, immune scores in stage iii/iv were also significantly 


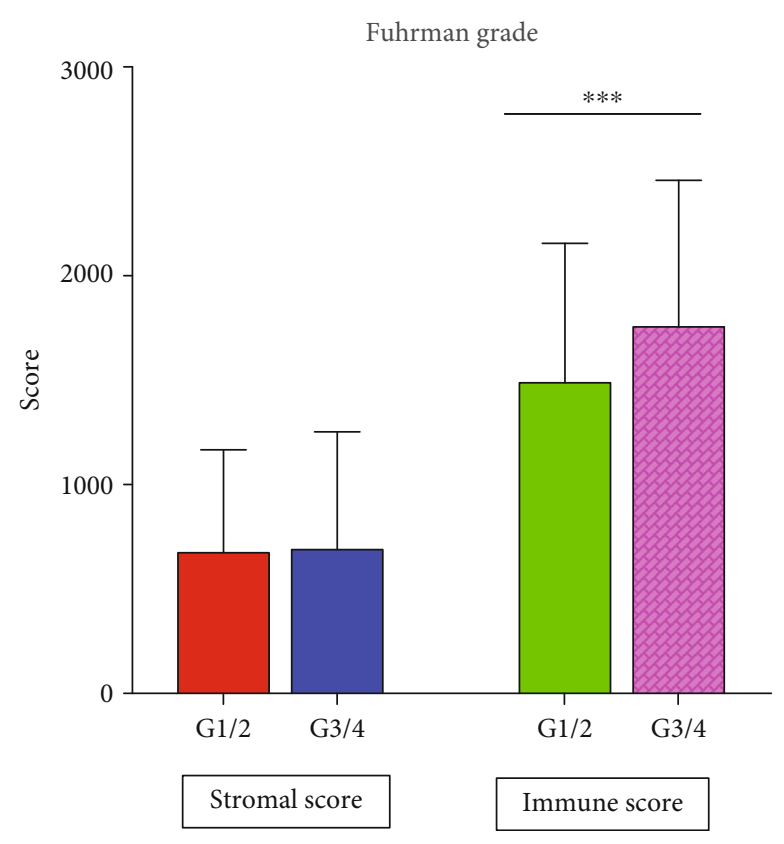

(a)

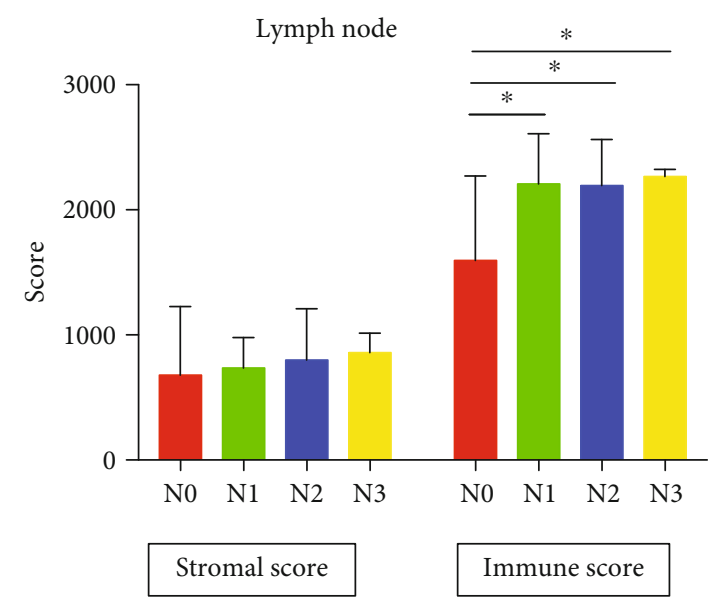

(c)

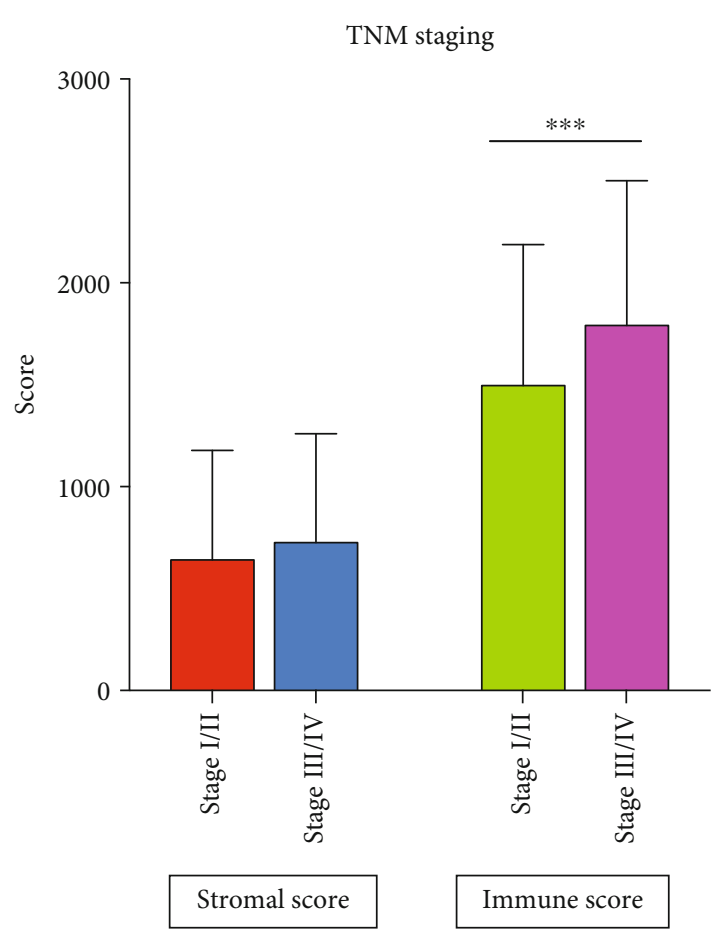

(b)

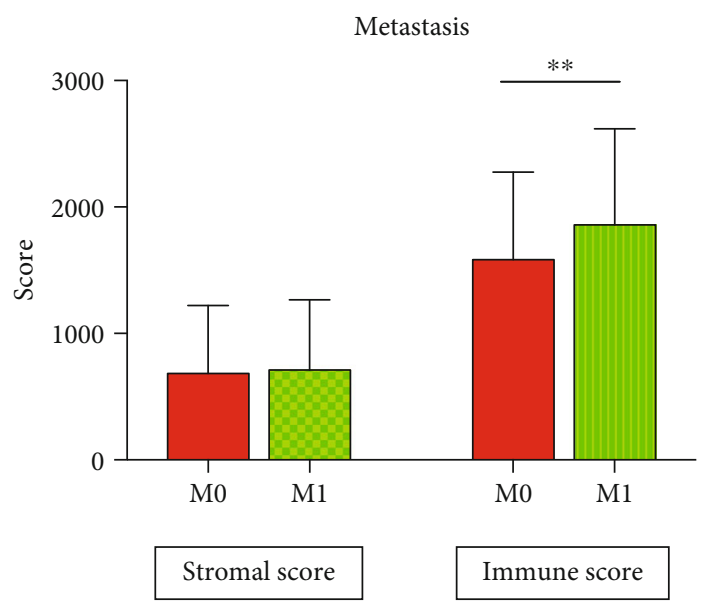

(d)

FIGURE 1: Distribution of stromal and immune scores. (a) Different Fuhrman grades and (b) TNM staging (c) with or without lymph node metastasis. N0, N1, N2, and N3 represent 0, 1, 2, and 3 lymph node metastases. (d) Distant metastasis.

increased (Figure 1(b), $P<0.001$ ). However, for stromal scores, there was no statistical difference between different Fuhrman grades $(P=0.738)$ and TNM staging $(P=0.083)$. When compared with patients without lymph node metastasis, patients with 1,2 , and 3 lymph node metastases had higher immune scores (Figure $1(\mathrm{c}), P=0.012, P=0.032$, and $P=0.016)$. Similar results were observed in patients with distant metastasis (Figure $1(\mathrm{~d}), P=0.001)$. Nevertheless, no difference of immune scores was observed between patients with or without lymph node metastasis $(P=0.529)$ and patients with or without distant metastasis $(P=0.685)$.

The associations of stromal/immune scores and corresponding overall survival were analyzed by the KaplanMeier plot and evaluated with the log-rank test. The
Kaplan-Meier plot demonstrated that high-immune scores as well as stromal scores were negatively correlated with favorable outcome of ccRCC patients (Figure 2). In addition, 5 -year survival rates of low- and high-immune score groups were $69.3 \%$ and $52.2 \%$, respectively $(\mathrm{HR}=1.659,95 \% \mathrm{CI}$ $[1.204,2.248])$. And, for low- and high-stromal score groups, the rates were $65.7 \%$ and $54.6 \%$, respectively $(\mathrm{HR}=1.409$, $95 \%$ CI $[1.024,1.938])$. In addition, the result of multivariable Cox regression indicated that both stromal and immune scores were independent prognostic factors (Table 2).

3.3. Functional Enrichment Analysis and PPI Network. A total of 89 overlapping DEGs were identified between lowand high-stromal/immune score groups, including 42 
Immune score
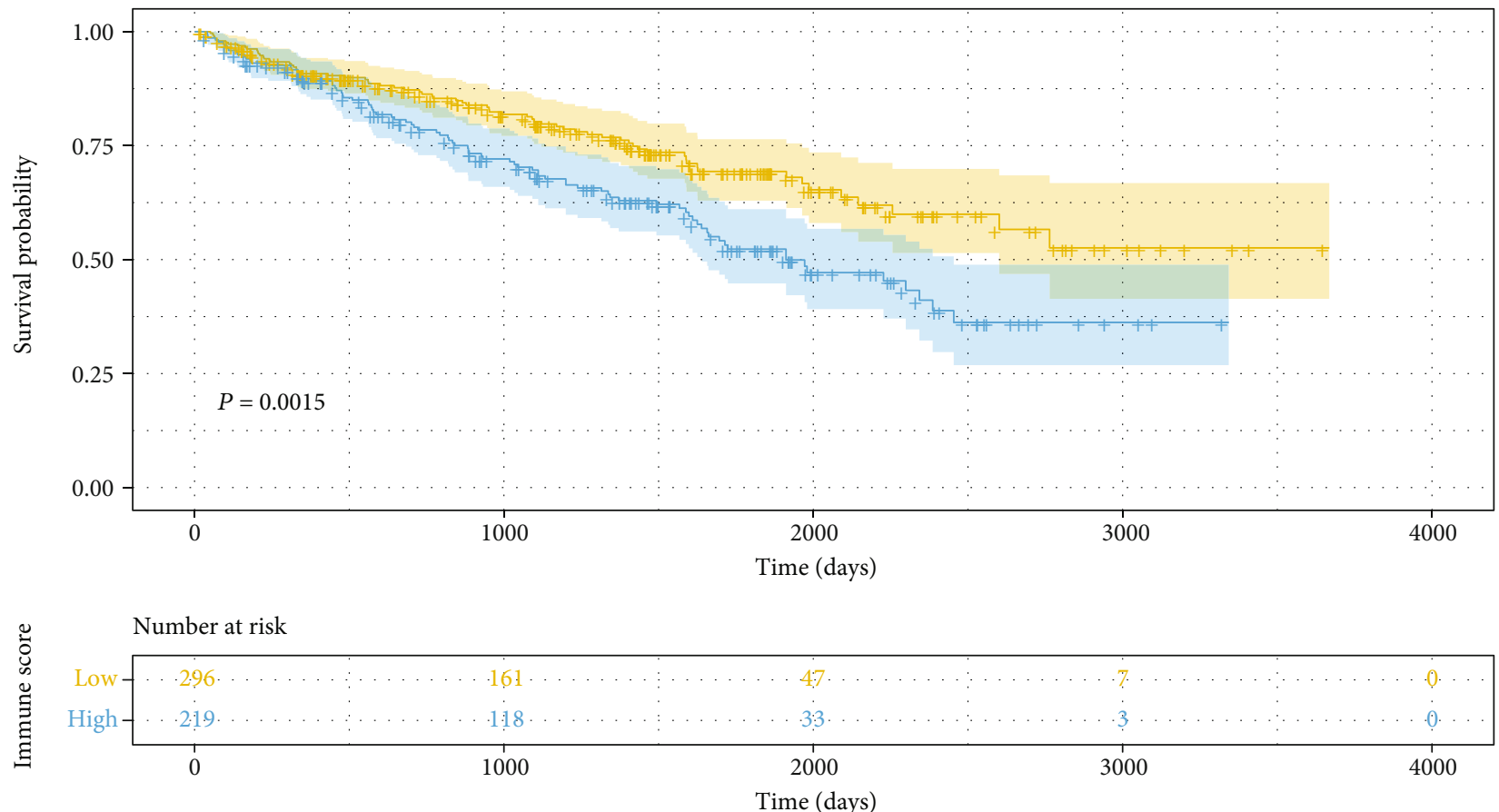

Low

(a)

Stromal score
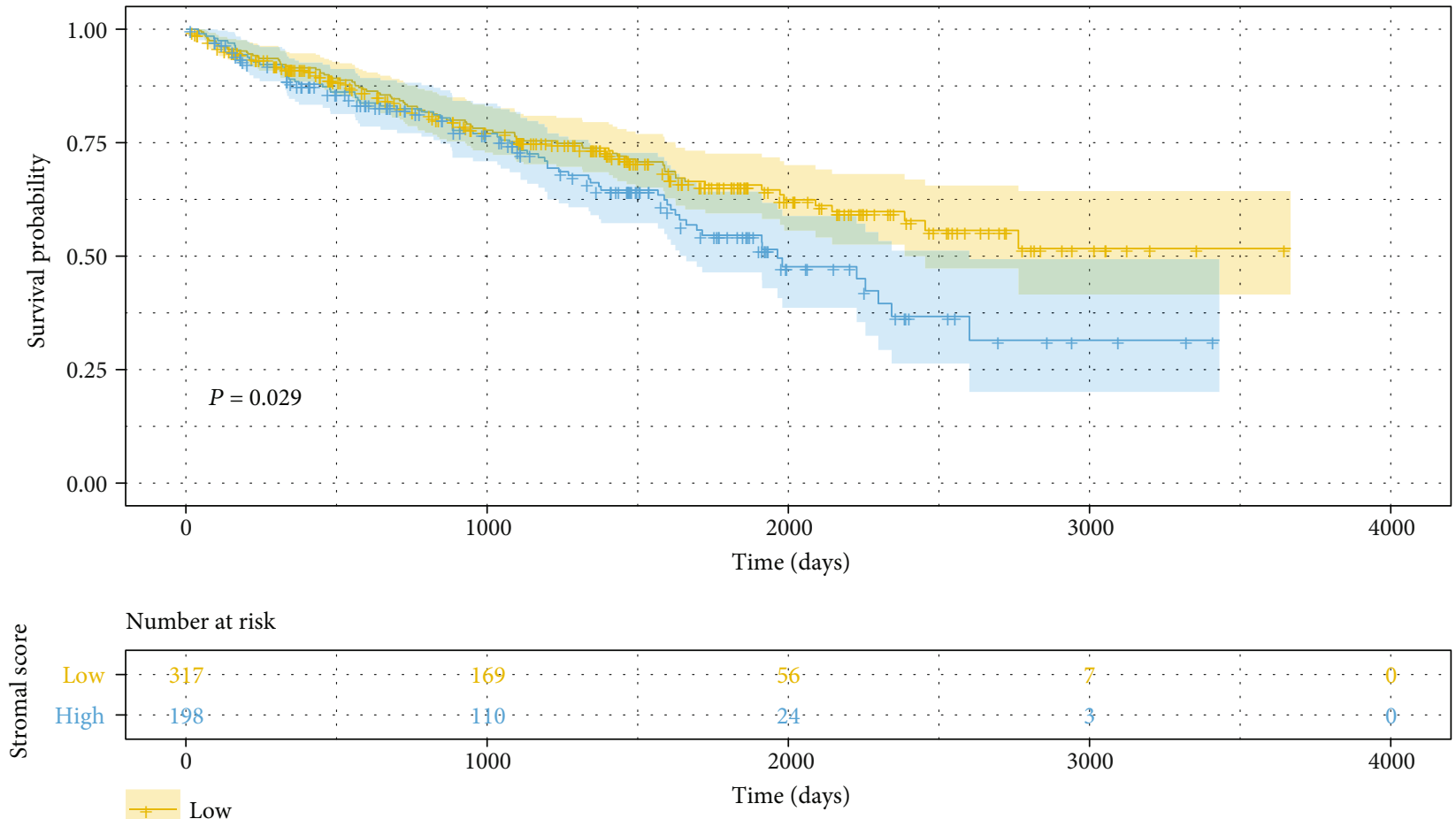

High

(b)

FIGURE 2: Prognostic values for overall survival: (a) immune scores and (b) stromal scores. 
TABLE 2: Results of univariate and multivariable Cox regression analysis.

\begin{tabular}{|c|c|c|c|c|c|c|}
\hline \multirow{2}{*}{ Characteristics } & \multicolumn{3}{|c|}{ Univariate analysis } & \multicolumn{3}{|c|}{ Multivariate analysis } \\
\hline & HR & $95 \% \mathrm{CI}$ & $P$ & HR & $95 \% \mathrm{CI}$ & $P$ \\
\hline Age $(<65 / \geq 65)$ & 1.690 & $(1.232,2.318)$ & 0.001 & 1.512 & $(1.096,2.087)$ & 0.012 \\
\hline Sex (female/male) & 0.937 & $(0.680,1.292)$ & 0.691 & 0.921 & $(0.665,1.275)$ & 0.619 \\
\hline Fuhrman grade ( 1 and $2 / 3$ and 4$)$ & 2.742 & $(1.923,3.911)$ & $<0.001$ & 1.771 & $(1.218,2.574)$ & 0.003 \\
\hline Stage (I and ii/iii and iv) & 4.361 & $(3.162,6.085)$ & $<0.001$ & 3.410 & $(2.394,4.857)$ & $<0.001$ \\
\hline Stromal scores (low/high) & 1.411 & $(1.134,1.925)$ & 0.030 & 1.236 & $(1.110,1.551)$ & 0.045 \\
\hline Immune scores (low/high) & 1.647 & $(1.207,2.248)$ & 0.002 & 1.321 & $(1.197,1.671)$ & 0.029 \\
\hline
\end{tabular}

HR: hazard ratio; 95\% CI: 95\% confidence interval.

upregulated and 47 downregulated overlapping genes (Figures 3(a) and 3(b)). To better understand the role of DEGs, KEGG and GO analyses were carried out. KEGG analysis revealed 89 overlapping DEGs which were mainly involved in 5 pathways, such as cytokine-cytokine receptor interaction, NF kappa B signaling pathway, and primary immunodeficiency (Figure 3(d)). In addition, 84 GO terms (67 terms of biological process, 1 term of cellular component, and 16 terms of molecular function) were enriched (Figure 3(e), Supplemental Table 1).

For exploring the interplay among 89 overlapping DEGs, a PPI network was built using the STRING tool with confidence $>0.7$ as the cut-off criterion. 24 nodes (21 upregulated and 3 downregulated DEGs) along with 39 edges consisted of the PPI network (Figure 3(c)). Moreover, in the PPI network, CD79a, CD19, CCL19, IL10, and XCR1 were the remarkable nodes as they had the most connections with other nodes. Furthermore, survival analysis revealed a significant correlation between the expression of IL10 (0.030) and XCR1 $(P=0.012)$ and prognosis of ccRCC (Supplemental Figure 1). In addition, the result showed that both IL10 and XCR1 were closely related to the infiltration of tumor immune cells (Supplemental Figure 2).

3.4. Expression Profile of Immunomodulators. As shown in Figure 4(a), all of 11 immunomodulators (LAG-3, TIM-3, CTLA-4, IFN- $\gamma$, ICOS, ICAM-1, TIGIT, PD-1, PDL-1, NKG2A, and VISTA) were significantly increased in ccRCC samples compared with normal kidney samples. Moreover, in the high-stromal score group, the expression of 11 immunomodulators was all upregulated (Figure 4(b)). In the high-immune score group, similar phenomenon was observed except for PD-1 and PDL-1 (Figure 4(c)). Then, we further explored the prognostic value of 11 immunomodulators. Survival analysis suggested that patients with low expression of LAG3 or CTLA-4 had a longer overall survival than those with high expression of LAG3 $(\mathrm{HR}=1.448,95 \%$ CI $[1.063,1.972]$, Figure $4(\mathrm{~d}))$ or CTLA-4 $(\mathrm{HR}=1.513,95 \%$ CI $[1.11,2.062]$, Figure 4(e)).

\section{Discussion}

ccRCC is one of the most prevalent kidney malignancies and accounts for approximately $3 \%$ of adult cancer [24, 25]. Previous studies have shown that the tumor microenvironment (TME) was highly related to the oncogenesis, progress, and prognosis of ccRCC [26]. TME is the place where the immune system and tumor interplay, reflecting the plasticity of both the tumor and immune system [27]. Tumor development is highly dependent on TME, and any alterations of the composition of TME may influence the evolution of malignancies [27]. Understanding the change may help the development of therapeutic strategies. Stromal cells and immune cells are the important components of TME, which play a critical role in the development of cancers [6]. The ESTIMATE algorithm, a tool based on a large scale of gene expression profile, has been used for the investigation of glioblastoma, colon cancer, and breast cancer and shown good precision and practicality [18-20]. However, it has not been applied for the research on ccRCC.

In the current study, we attempted to infer the level of infiltrating stromal and immune cells in ccRCC by calculating stromal and immune scores with the ESTIMATE algorithm. Survival curves according to Kaplan-Meier showed that low-immune scores as well as stromal scores predicted a favorable prognosis in ccRCC patients. In addition, evidence indicating a significant increase in immune scores was witnessed in patients in Fuhrman grade $3 / 4$ and stage iii/iv, with lymph node metastasis as well as with distant metastasis, whereas for stromal scores, no differences were observed between different Fuhrman grades and TNM staging, with or without lymph node metastasis or distant metastasis.

For exploring the potential mechanism of the change of TME, we identified tumor microenvironment-related genes which would be further utilized for functional enrichment analysis and constructing the PPI network. A total of 89 overlapping differentially expressed genes (DEGs) (42 upregulated and 47 downregulated genes) were determined. KEGG analysis demonstrated that 5 pathways were enriched by 89 overlapping DEGs, including cytokine-cytokine receptor interaction, NF kappa B signaling pathway, and primary immunodeficiency. The NF kappa B signaling pathway is involved in immunity, inflammation, and cell survival [28]. Lua et al. found that the NF kappa B signaling pathway could affect the prognosis of RCC by decreasing the local inflammatory infiltrate and regulating TME [29]. Additionally, Morais et al. reported that inhibition of the NF kappa B signaling pathway attenuated the progression of RCC [30]. Meanwhile, the PPI network was constructed to predict the interaction relationship among 89 overlapping DEGs, and the top 5 degree genes (CD79a, CD19, CCL19, IL10, and 


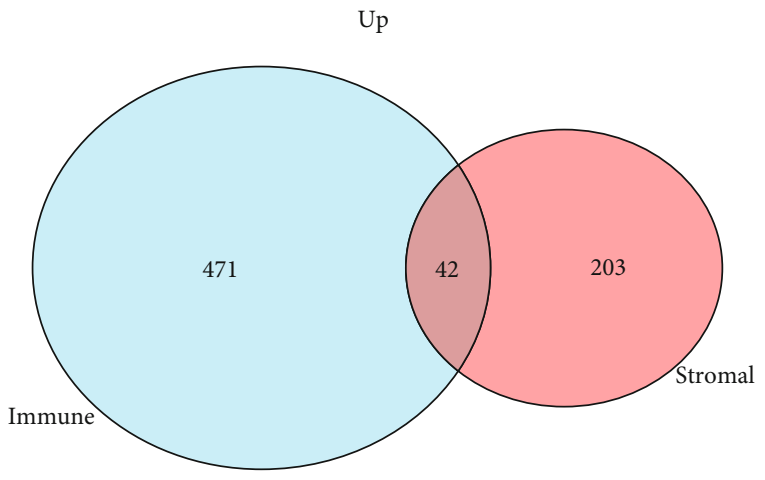

(a)

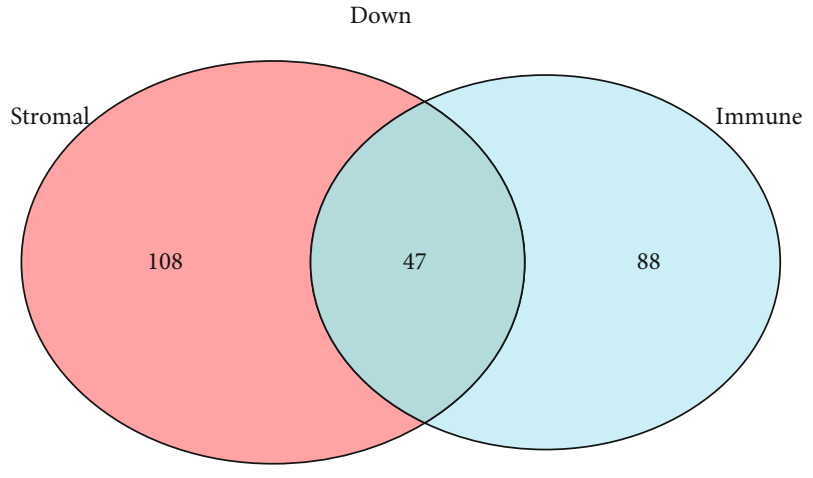

(b)

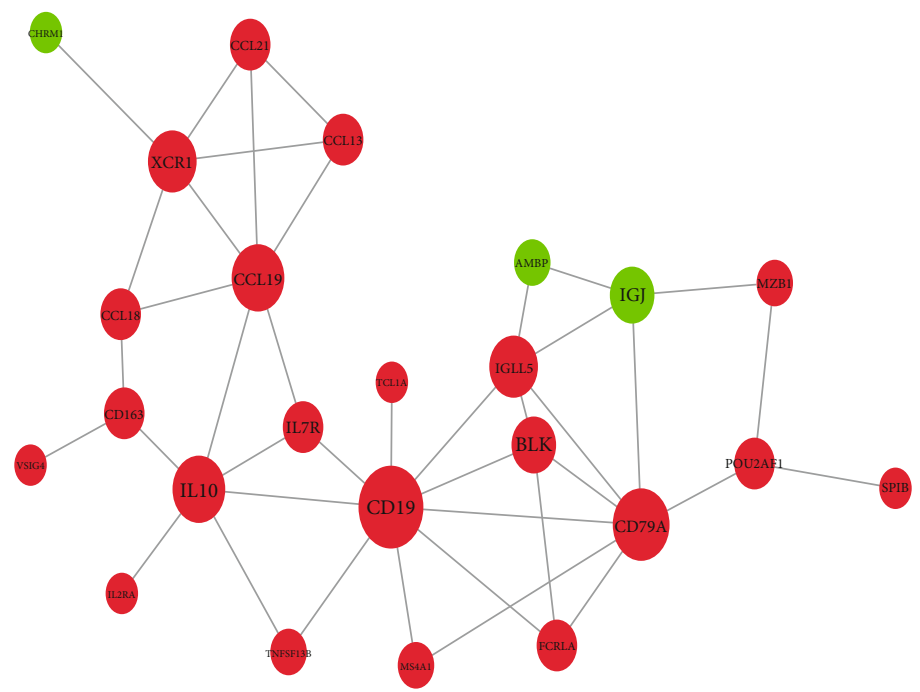

(c)

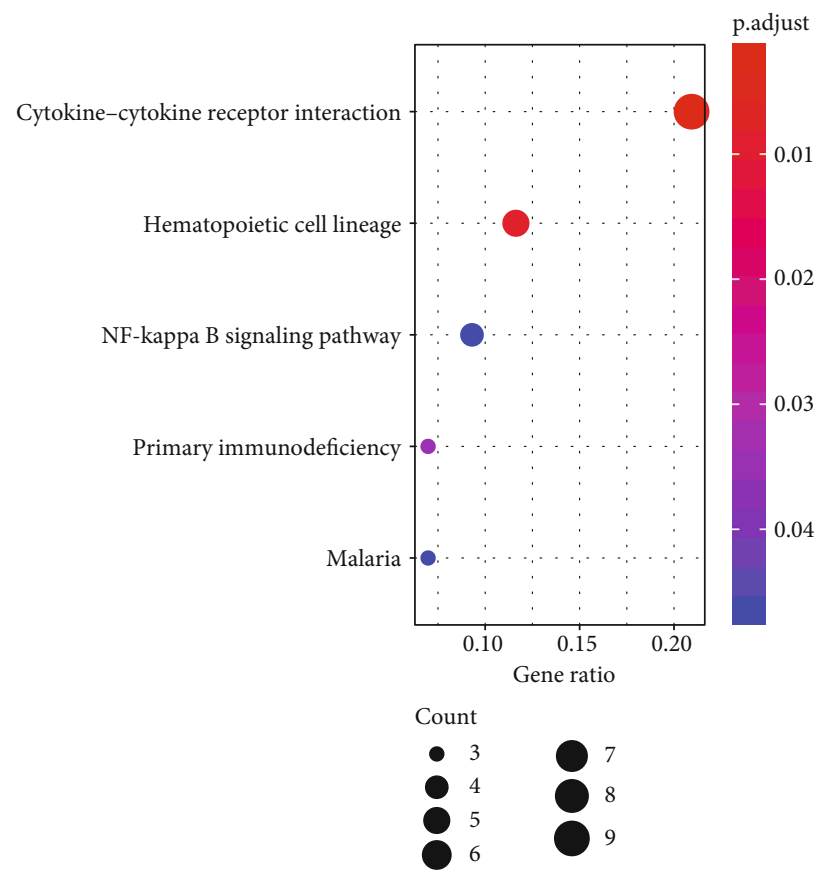

(d)

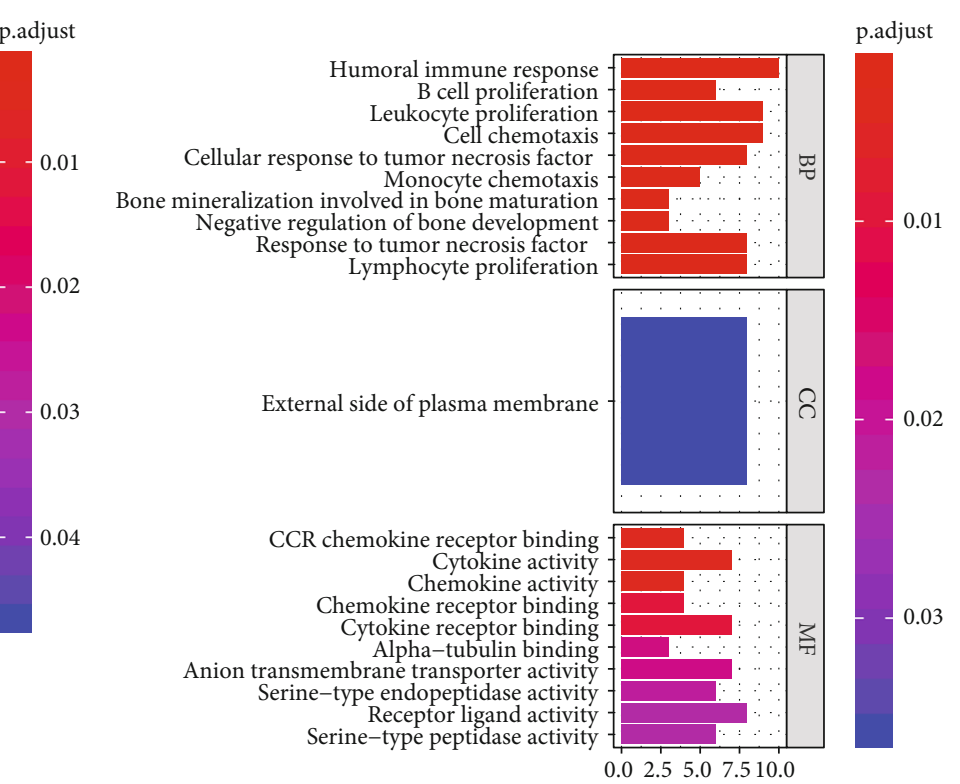

(e)

Figure 3: Analysis of DEGs. (a) 42 upregulated overlapping DEGs and (b) 47 downregulated overlapping DEGs. (c) Protein-protein interaction (PPI) network with confidence $>0.7$. Red and green nodes represent upregulated and downregulated genes, respectively. (d) The enriched pathways with $P<0.05$. (e) The enriched GO terms with $P<0.05$ and gene count $>5$. 


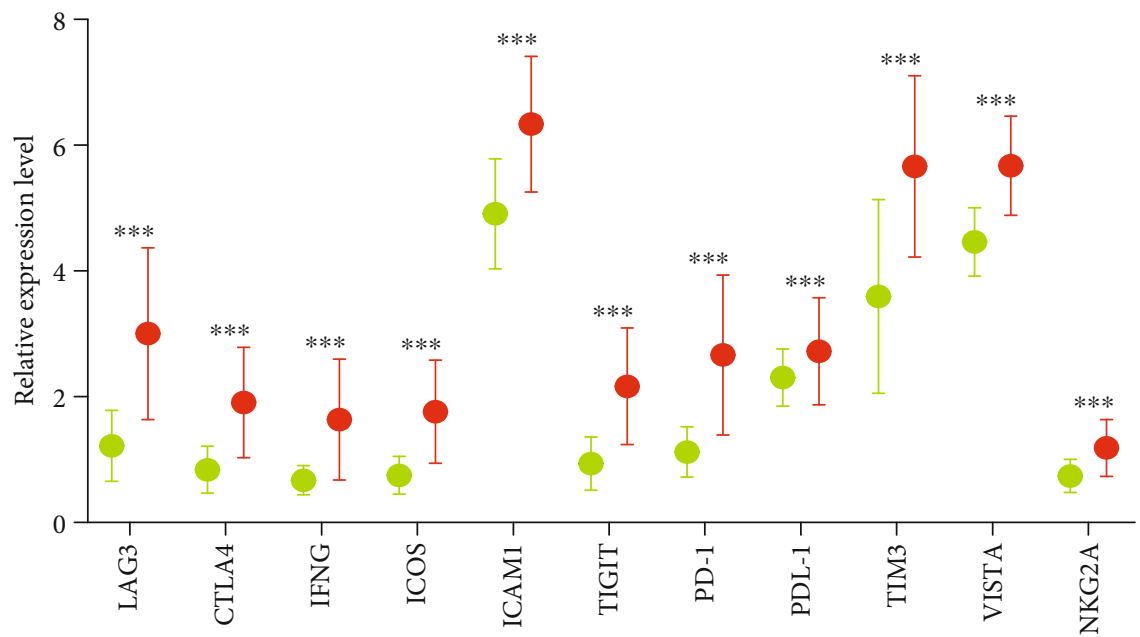

Normal

- Cancer

(a)

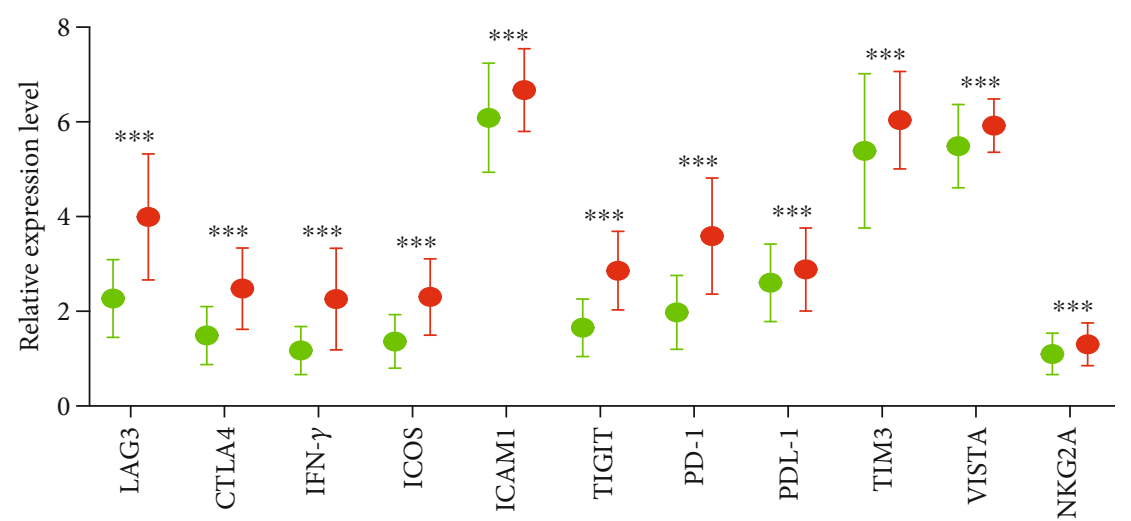

Low immune score

- High immune score

(b)

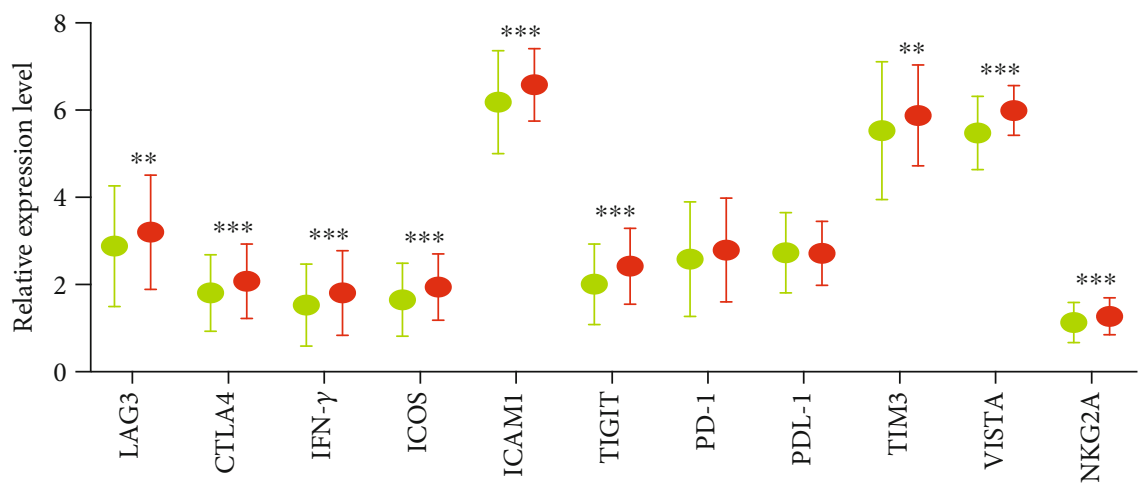

Low stromal score

High stromal score

(c)

FIgure 4: Continued. 
CTLA4
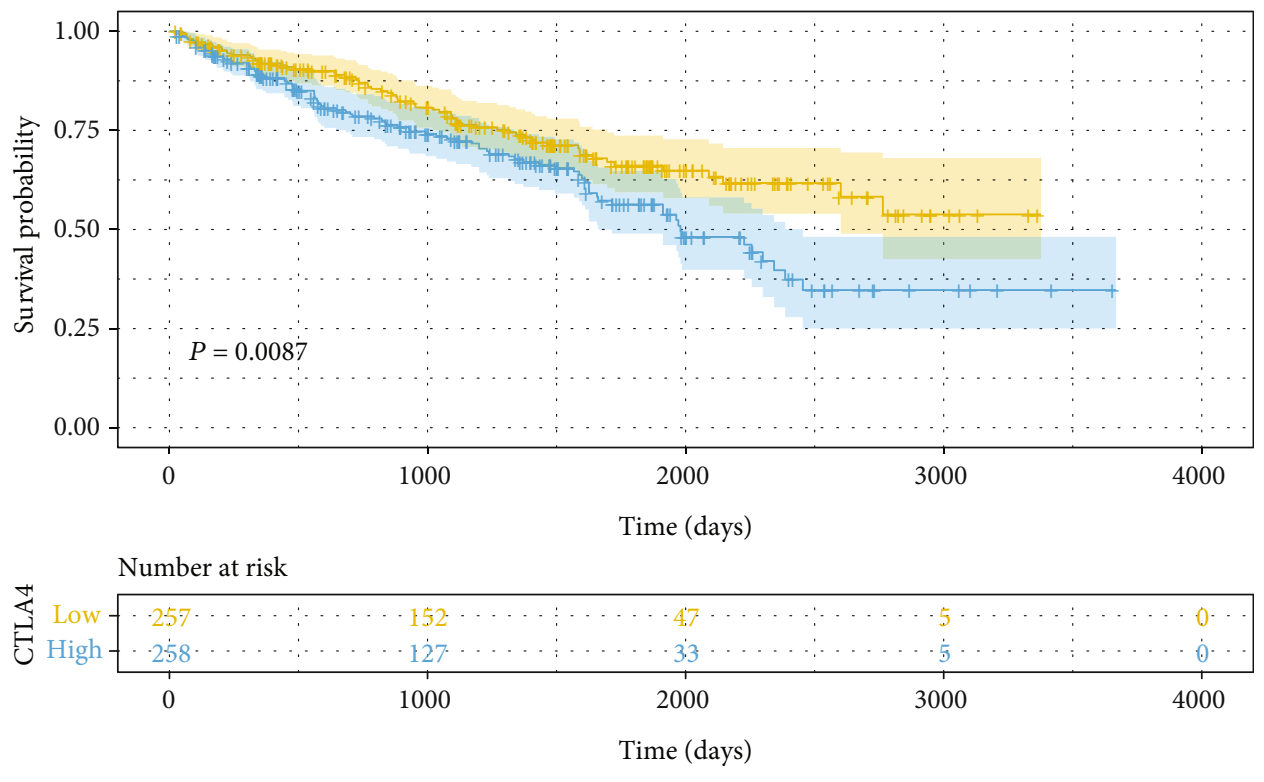

+ Low
$+\quad$ High

(d)

LAG3

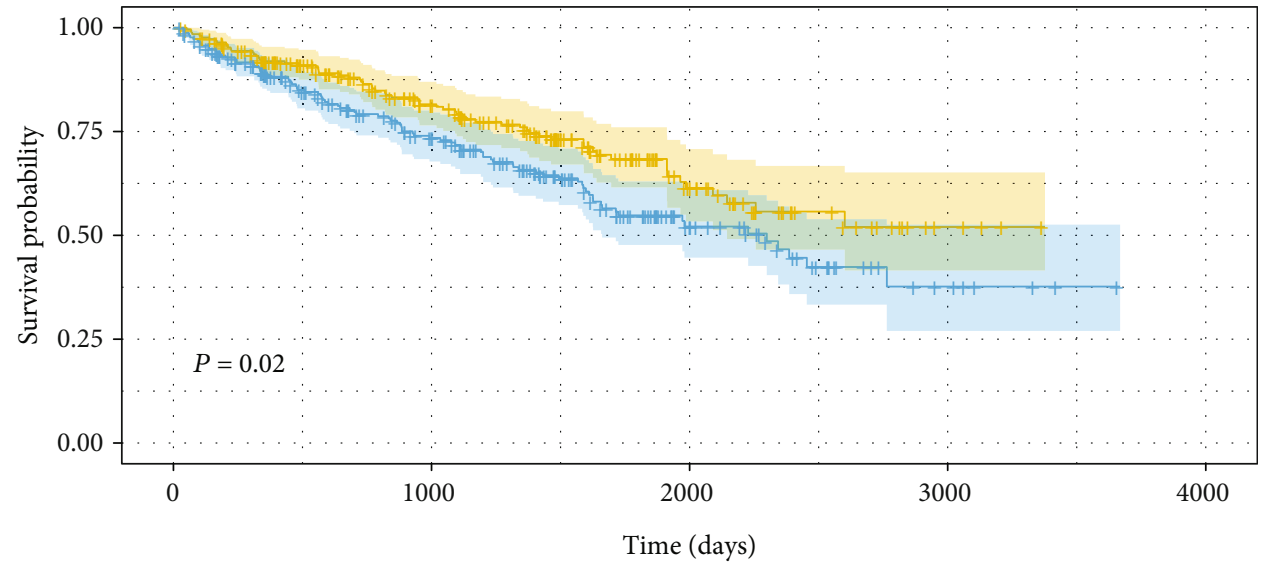

Number at risk

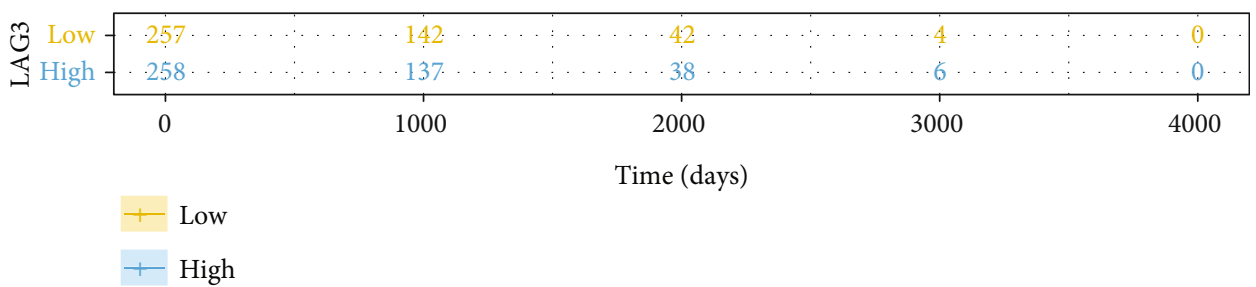

(e)

FIgURE 4: The distribution of immunomodulators (a) between normal samples and ccRCC samples, (b) low- and high-immune scores, and (c) low- and high-stromal scores. (d) Kaplan-Meier curves for overall survival of CTLA-4 and (e) LAG-3.

XCR1) were extracted for further analysis. Among the top 5 degree genes, 2 DEGs (IL10 and XCR1) were highly relative to clinical outcome of ccRCC patients as well as the infiltration of tumor immune cells. IL10, a cytokine produced by monocytes and lymphocytes primarily, has pleiotropic effects on regulating immune response by stimulating $\mathrm{B}$ cells and inhibiting macrophages and helper $\mathrm{T}$ cells [31]. A previous study showed that the RCC patients with high expression of IL10 had a lower incidence of distant metastasis [32]. However, another research suggested that IL10-producing B cells 
were higher in advanced-stage RCC and could decrease the proportion of T cells in TME [33]. XCR1, the only receptor of the chemokine XCL1, is expressed in dendritic cells and has an implicated function in dendritic cell-mediated cytotoxic immune response [34]. Besides, the overexpression of $\mathrm{XCR} 1$ can promote the growth, migration, and invasion in breast cancer and non-small-cell lung cancer $[35,36]$. All data suggested that IL10 and XCR1 may be potential key regulators for the TME of ccRCC and novel markers for the prognosis of ccRCC.

Recently, immune checkpoint inhibitors have evolved treatment strategies in oncology and have been approved for the therapy of various cancers including RCC [23]. In this study, we determined 11 immunomodulators that are involved in tumor escape mechanisms and found 9 immunomodulators (LAG-3, TIM-3, CTLA-4, IFN- $\gamma$, ICOS, ICAM1, TIGIT, NKG2A, and VISTA) that were upregulated in both high-immune score group and high-stromal score group. In addition, PD-1 and PDL-1 were also increased in the high-stromal score group. Kaplan-Meier analysis demonstrated that longer overall survival among patients with low expression of LAG-3 and CTLA-4 was observed. CTLA-4, a homolog of CD28, is expressed by T cells and could inhibit the $\mathrm{T}$ cell immune response by diminishing costimulatory signal [37]. LAG-3 is expressed in activated $\mathrm{CD} 4+$ and $\mathrm{CD} 8+\mathrm{T}$ cells and participates in helper $\mathrm{T}$ cell response [38]. Several researches have shown that LAG-3 expression was related to the metastasis and prognosis of various cancers such as breast cancer, lung cancer, and ovarian cancer [39-41].

There are a few limitations to be addressed in this study. Firstly, due to the fact that all patients were gathered from TCGA database, the potential of selection bias could not be excluded and it was not possible to collect all information of patients, such as the organ distributions with metastasis and information on whether to use anti-inflammatory drugs or not. Secondly, there was no experimental research conducted to examine the functions of IL10 and XCR1 in ccRCC. Thus, further investigation both in vitro and in vivo is demanded to testify the discovery of this research.

In summary, we applied the ESTIMATE algorithm to calculate stromal and immune scores which were highly associated with the clinical outcome of ccRCC. In addition, we identified 89 microenvironment-related genes, and data from the PPI network and survival analysis revealed that IL10 and XCR1 may the potential key regulators for the TME of ccRCC and could be useful for outlining the prognosis of ccRCC patients. However, more experimental research both in vitro and in vivo is needed to examine the finding of this research.

\section{Data Availability}

The data used to support the findings of this study are included within the article.

\section{Conflicts of Interest}

The authors declare that there is no conflict of interests.

\section{Authors' Contributions}

$\mathrm{YH}$ and $\mathrm{YF}$ conceived and designed the study. WC and BC performed the majority of the experiments and wrote the paper. JJ and XW analyzed the experimental data. YH and YF reviewed and edited the manuscript. All authors approved the manuscript and agree to be accountable for all aspects of the research in ensuring that the accuracy or integrity of any part of the work is appropriately investigated and resolved. Bin Chen and Wei Chen contributed equally to this work.

\section{Acknowledgments}

This work was supported by the Jiaxing Science and Technology Project (2018AD32091 and 2018AD32083) and the Key Subject of Jiaxing Medicine (2019-zc-07).

\section{Supplementary Materials}

Supplemental Table 1 Gene Ontology analysis of 89 overlapping DEGs. Supplemental Figure 1: prognostic values for overall survival. (A) IL10. (B) XCR1. Supplemental Figure 2: the relationship between hub genes and tumorinfiltrating immune cells (B cell, CD4 T cell, CD8 T cell, neutrophil, macrophage, and dendritic cell). (Supplementary Materials)

\section{References}

[1] K. Yang, X. F. Lu, P. C. Luo, and J. Zhang, "Identification of six potentially long noncoding RNAs as biomarkers involved competitive endogenous RNA in clear cell renal cell carcinoma," BioMed Research International, vol. 2018, Article ID 303486, 13 pages, 2018.

[2] U. Capitanio and F. Montorsi, "Renal cancer," The Lancet, vol. 387, no. 10021, pp. 894-906, 2016.

[3] T. Xing and H. He, "Epigenomics of clear cell renal cell carcinoma: mechanisms and potential use in molecular pathology," Chinese journal of cancer research, vol. 28, no. 1, pp. 80-91, 2016.

[4] J. Godlewski, J. Kiezun, B. E. Krazinski, Z. Kozielec, P. M. Wierzbicki, and Z. Kmiec, "The Immunoexpression of YAP1 and LATS1 Proteins in Clear Cell Renal Cell Carcinoma: Impact on Patients' Survival," BioMed Research International, vol. 2018, Article ID 2653623, 9 pages, 2018.

[5] C. Roma-Rodrigues, R. Mendes, P. V. Baptista, and A. R. Fernandes, "Targeting tumor microenvironment for cancer therapy," International Journal of Molecular Sciences, vol. 20, no. 4, p. 840, 2019.

[6] Y. Xiong, K. Wang, H. Zhou, L. Peng, W. You, and Z. Fu, "Profiles of immune infiltration in colorectal cancer and their clinical significant: A gene expression-based study," Cancer Medicine, vol. 7, no. 9, pp. 4496-4508, 2018.

[7] Z. Liu, Y. Zhu, J. Z. Le Xu et al., "Tumor stroma-infiltrating mast cells predict prognosis and adjuvant chemotherapeutic benefits in patients with muscle invasive bladder cancer," OncoImmunology, vol. 7, no. 9, article e1474317, 2018.

[8] J. Wu, C. Liang, M. Chen, and W. Su, "Association between tumor-stroma ratio and prognosis in solid tumor patients: a 
systematic review and meta-analysis," Oncotarget, vol. 7, no. 42, pp. 68954-68965, 2016.

[9] B. Youngblood, J. S. Hale, H. T. Kissick et al., "Effector CD8 T cells dedifferentiate into long-lived memory cells," Nature, vol. 552, no. 7685, pp. 404-409, 2017.

[10] J. Yao, W. Xi, Y. Zhu, H. Wang, X. Hu, and J. Guo, "Checkpoint molecule $\mathrm{PD}-1$-assisted $\mathrm{CD} 8<$ sup $>+</$ sup $>$ T lymphocyte count in tumor microenvironment predicts overall survival of patients with metastatic renal cell carcinoma treated with tyrosine kinase inhibitors," Cancer management and research, vol. 10, pp. 3419-3431, 2018.

[11] Y. Wang, Y. Wang, L. Xu et al., "CD4 + T cells promote renal cell carcinoma proliferation _via_modulating YBX1," Experimental Cell Research, vol. 363, no. 1, pp. 95-101, 2018.

[12] D. E. Speiser, P. C. Ho, and G. Verdeil, "Regulatory circuits of T cell function in cancer," Nature Reviews Immunology, vol. 16, no. 10, pp. 599-611, 2016.

[13] T. Motoshima, Y. Miura, N. Wakigami et al., "Phenotypical change of tumor-associated macrophages in metastatic lesions of clear cell renal cell carcinoma," Medical Molecular Morphology, vol. 51, no. 1, pp. 57-63, 2018.

[14] G. C. Hutterer, M. Pichler, T. F. Chromecki et al., “Tumourassociated macrophages might represent a favourable prognostic indicator in patients with papillary renal cell carcinoma," Histopathology, vol. 63, no. 3, pp. 309-315, 2013.

[15] N. Rohr-Udilova, F. Klinglmüller, R. Schulte-Hermann et al., "Deviations of the immune cell landscape between healthy liver and hepatocellular carcinoma," Scientific Reports, vol. 8, no. 1, p. 6220, 2018.

[16] R. Zhou, J. Zhang, D. Zeng et al., "Immune cell infiltration as a biomarker for the diagnosis and prognosis of stage I-III colon cancer," Cancer Immunology, Immunotherapy, vol. 68, no. 3, pp. 433-442, 2019.

[17] K. Yoshihara, M. Shahmoradgoli, E. Martínez et al., "Inferring tumour purity and stromal and immune cell admixture from expression data," Nature Communications, vol. 4, no. 1, p. 2612, 2013.

[18] D. Jia, S. Li, D. Li, H. Xue, D. Yang, and Y. Liu, "Mining TCGA database for genes of prognostic value in glioblastoma microenvironment," Aging, vol. 10, no. 4, pp. 592-605, 2018.

[19] M. H. Alonso, S. Aussó, A. Lopez-Doriga et al., "Comprehensive analysis of copy number aberrations in microsatellite stable colon cancer in view of stromal component," British Journal of Cancer, vol. 117, no. 3, pp. 421-431, 2017.

[20] N. Priedigkeit, R. J. Watters, P. C. Lucas et al., "Exome-capture RNA sequencing of decade-old breast cancers and matched decalcified bone metastases," JCI Insight, vol. 2, no. 17, p. 95703, 2017.

[21] J. Budczies, F. Klauschen, B. V. Sinn et al., "Cutoff Finder: a comprehensive and straightforward web application enabling rapid biomarker cutoff optimization," PLoS One, vol. 7, no. 12, article e51862, 2012.

[22] D. Szklarczyk, A. L. Gable, D. Lyon et al., "STRING v11: protein-protein association networks with increased coverage, supporting functional discovery in genome-wide experimental datasets," Nucleic Acids Research, vol. 47, no. D1, pp. D607D613, 2019.

[23] S. Sadreddini, B. Baradaran, A. Aghebati-Maleki et al., "Immune checkpoint blockade opens a new way to cancer immunotherapy," Journal of Cellular Physiology, vol. 234, no. 6, pp. 8541-8549, 2019.
[24] S. J. D. Vecchio and R. J. Ellis, "Cabozantinib for the management of metastatic clear cell renal cell carcinoma," Journal of Kidney Cancer and VHL, vol. 5, no. 4, pp. 1-5, 2018.

[25] W. Majer, K. Kluzek, H. Bluyssen, and J. Wesoły, "Potential approaches and recent advances in biomarker discovery in clear-cell renal cell carcinoma," Journal of Cancer, vol. 6, no. 11, pp. 1105-1113, 2015.

[26] S. Zhang, E. Zhang, J. Long et al., "Immune infiltration in renal cell carcinoma," Cancer Science, vol. 110, no. 5, pp. 1564-1572, 2019.

[27] M. C. Merlano, A. Abbona, N. Denaro, and O. Garrone, "Knowing the tumour microenvironment to optimise immunotherapy," Acta Otorhinolaryngologica Italica, vol. 39, no. 1, pp. 2-8, 2019.

[28] C. Morais, G. Gobe, D. W. Johnson, and H. Healy, "The emerging role of nuclear factor kappa B in renal cell carcinoma," The International Journal of Biochemistry \& Cell Biology, vol. 43, no. 11, pp. 1537-1549, 2011.

[29] J. Lua, T. Qayyum, J. Edwards, and A. K. Roseweir, "The prognostic role of the non-canonical nuclear factor-kappa B pathway in renal cell carcinoma patients," Urologia Internationalis, vol. 101, no. 2, pp. 190-196, 2018.

[30] C. Morais, H. Healy, D. W. Johnson, and G. Gobe, "Inhibition of nuclear factor kappa B attenuates tumour progression in an animal model of renal cell carcinoma," Nephrology Dialysis Transplantation, vol. 25, no. 5, pp. 1462-1474, 2010.

[31] C. Burrello, F. Garavaglia, F. M. Cribiù et al., "Therapeutic faecal microbiota transplantation controls intestinal inflammation through IL10 secretion by immune cells," Nature Communications, vol. 9, no. 1, p. 5184, 2018.

[32] N. Uwatoko, T. Tokunaga, H. Hatanaka et al., "Expression of interleukin-10 is inversely correlated with distant metastasis of renal cell carcinoma," International Journal of Oncology, vol. 20, no. 4, pp. 729-733, 2002.

[33] C. Cai, J. Zhang, M. Li et al., "Interleukin 10-expressing B cells inhibit tumor-infiltrating $\mathrm{T}$ cell function and correlate with $\mathrm{T}$ cell Tim-3 expression in renal cell carcinoma," Tumor Biology, vol. 37, no. 6, pp. 8209-8218, 2016.

[34] M. G. Serrán, F. F. Vernengo, C. G. Beccaria, E. V. Acosta Rodriguez, C. L. Montes, and A. Gruppi, "The regulatory role of B cells in autoimmunity, infections and cancer: perspectives beyond IL10 production," FEBS Letters, vol. 589, no. 22, pp. 3362-3369, 2015.

[35] X. L. Yang, L. G. Qi, F. J. Lin, and Z. L. Ou, "The role of the chemokine receptor XCR1 in breast cancer cells," Breast Cancer: Targets and Therapy, vol. 9, pp. 227-236, 2017.

[36] T. Wang, S. Han, Z. Wu et al., "XCR1 promotes cell growth and migration and is correlated with bone metastasis in nonsmall cell lung cancer," Biochemical and Biophysical Research Communications, vol. 464, no. 2, pp. 635-641, 2015.

[37] A. Bertrand, M. Kostine, T. Barnetche, M. E. Truchetet, and T. Schaeverbeke, "Immune related adverse events associated with anti-CTLA-4 antibodies: systematic review and metaanalysis," BMC Medicine, vol. 13, no. 1, p. 211, 2015.

[38] L. P. Andrews, A. E. Marciscano, C. G. Drake, and D. A. Vignali, "LAG3 (CD223) as a cancer immunotherapy target," Immunological Reviews, vol. 276, no. 1, pp. 80-96, 2017.

[39] S. M. Hald, M. Rakaee, I. Martinez et al., "LAG-3 in NonSmall-cell Lung Cancer: Expression in Primary Tumors and 
Metastatic Lymph Nodes Is Associated With Improved Survival," Clinical Lung Cancer, vol. 19, no. 3, pp. 249-259.e2, 2018.

[40] S. Burugu, D. Gao, S. Leung, S. K. Chia, and T. O. Nielsen, "LAG-3+ tumor infiltrating lymphocytes in breast cancer: clinical correlates and association with PD-1/PD-L1+ tumors," Annals of Oncology, vol. 28, no. 12, pp. 2977-2984, 2017.

[41] R.-Y. Huang, A. Francois, A. J. R. McGray, A. Miliotto, and K. Odunsi, "Compensatory upregulation of PD-1, LAG-3, and CTLA-4 limits the efficacy of single-agent checkpoint blockade in metastatic ovarian cancer," OncoImmunology, vol. 6, no. 1, article e1249561, 2016. 


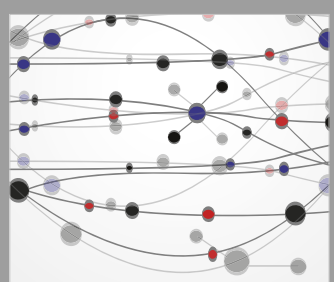

The Scientific World Journal
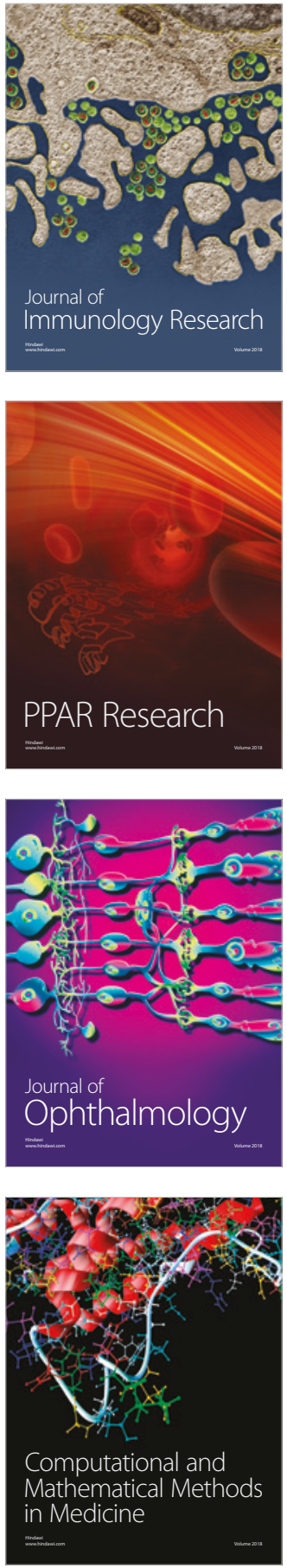

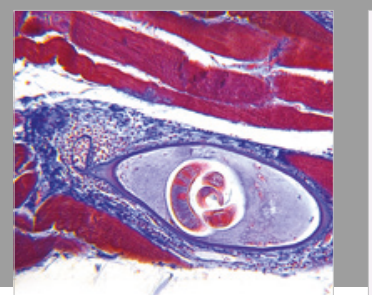

Gastroenterology Research and Practice

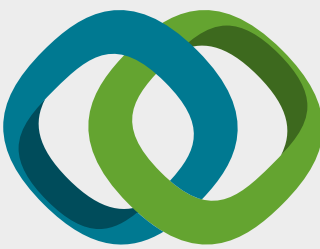

\section{Hindawi}

Submit your manuscripts at

www.hindawi.com
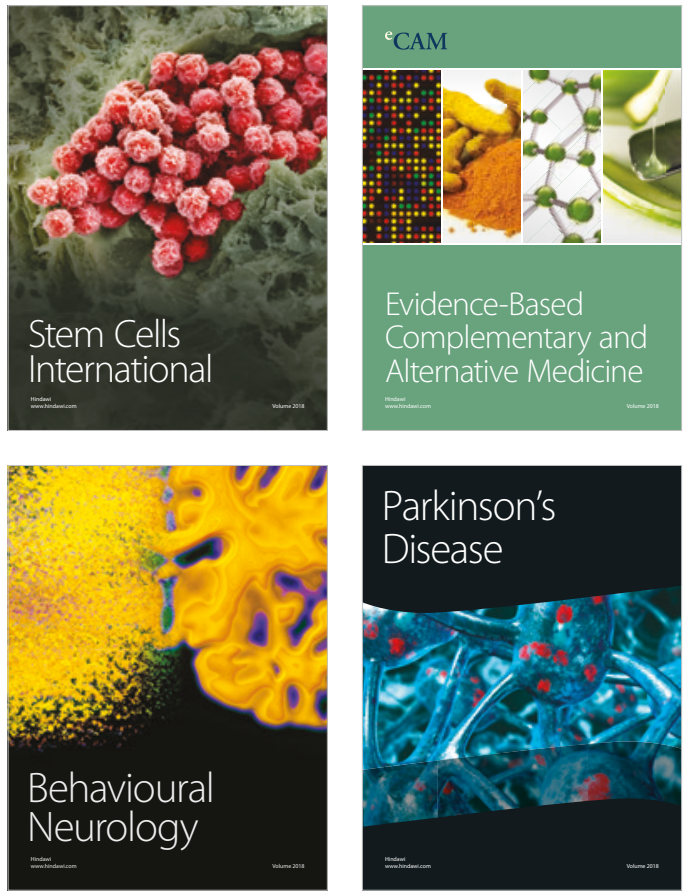

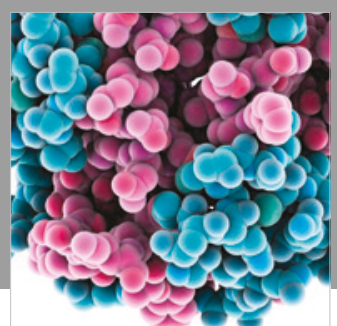

ournal of

Diabetes Research

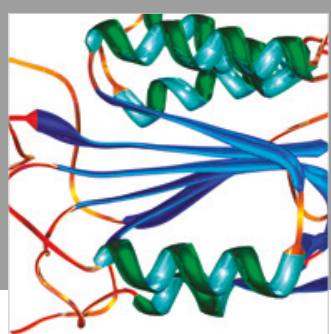

Disease Markers
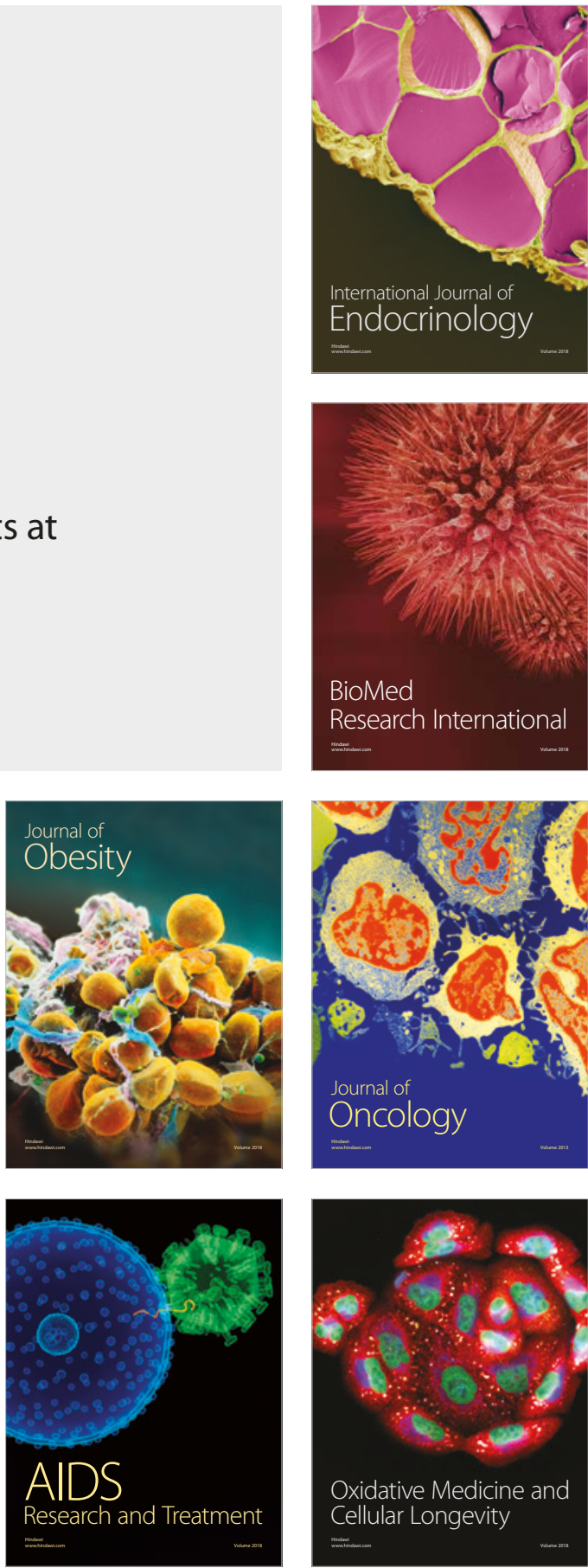